\title{
Transition d'un système de carrière à système d'emploi au travers d'un modèle hybride (Modernisation de la fonction publique en Hongrie)
}

\author{
Zoltan Hazafi \\ Université Nationale de Service Public; Budapest, Hongrie \\ drhazafi@gmail.com
}

RÉSUMÉ

\begin{abstract}
La modernisation de la fonction publique hongroise, démarrée en 2010, se fonde sur une approche stratégique et complexe ainsi que sur un compromis entre les anciennes et les nouvelles valeurs. L'objectif stratégique est d'une part, le développement d'une carrière prévisible et attractive, et d'autre part, la mise en place d'une gestion des ressources humaines basée sur l'emploi. À première vue, les valeurs du modèle de carrière et celles de la gestion moderne des ressources humaines pourraient paraître contradictoires. Cependant, le système hybride caractérisant le régime juridique de la fonction publique hongroise peut combiner ces différentes valeurs en mettant l'emploi au centre de la gestion des ressources humaines. Par ailleurs, "l'hybridation " est non seulement l'une des spécificités de la fonction publique hongroise mais aussi celle de l'évolution du contexte international.
\end{abstract}

Mots-clés: système hybride, système d'emploi, gestion des ressources humaines, fonction publique hongroise, fragmentation

JEL: M54

\section{Introduction}

\subsection{Contexte international}

De nos jours, l'évolution de la fonction publique est caractérisée au moins par deux phénomènes. D'une part, les pays dotés d'une fonction publique à carrière se préoccupent souvent d'adopter dans leur fonction publique les techniques typiques du secteur privé, et de rendre plus flexibles les conditions de travail (rémunération en fonction de la performance, heures de travail flexibles, etc.). D'autre part, les gouvernements exigent non seulement une plus grande flexibilité et une meilleure efficacité, mais favorisent également le renforcement des valeurs wébériennes comme l'intégrité, la régularité, le professionnalisme, la non-discrimination ainsi que l'aversion au risque. 
La question se pose régulièrement de savoir si la législation traditionnelle représentant les valeurs bureaucratiques basées sur les carrières peut être mise en conformité avec les processus de RH imprégnés de valeurs managériales qui placent l'emploi au centre de la gestion des ressources humaines. Selon certains auteurs, les deux facteurs peuvent évolver en s'adaptant l'un à l'autre ou en se distinguant l'un de l'autre, voire dans des directions opposées (van der Meer, van der Berg et Dijkstra, 2013, pp. 95-113). Les valeurs traditionnelles et la gestion moderne des ressources humaines ne se contredisent pas, car si les premières fixent les cadres, la deuxième détermine les processus, les outils et les méthodologies à utiliser à l'intérieur de ces cadres (Moniolle, 2010, pp. 234-240).

L'emploi contractuel est étroitement lié à l'importance croissante du droit du travail et du nouveau management public (NPM) qui ont mis à l'ordre du jour la révision de la réglementation traditionnelle du droit public dans plusieurs pays européens. Cela peut même être considéré comme une caractéristique commune parmi ces pays. D'après certains auteurs, on peut différencier les pays selon les réponses qu'ils ont apportées aux questions posées par la flexibilisation (van der Meer et al., 2013, pp. 95-113). Certains pays visent à maintenir le statu quo actuel où, même si des mesures ont été prises pour l'introduction des outils de RH permettant une plus grande flexibilité, les réformes mises en œuvre n'ont pas dépassé les cadres traditionnels du droit public. C'est le cas, entre autres, en Allemagne, en Belgique et en France. Dans d'autres pays, on peut observer un retrait de la réglementation spécifique. C'est le cas des pays scandinaves, de l'ttalie et de la Suisse. Et, finalement, on peut distinguer les pays qui ont été particulièrement ambitieux ces dernières années, et ont mis en place ou renforcé la réglementation wébérienne. Ce sont les pays d'Europe centrale et orientale, et la Grande-Bretagne.

À mon avis, cette catégorisation semble un peu trop simpliste, et l'analyse de la situation devrait être nuancée. En ce qui concerne la sauvegarde des cadres traditionnels du droit public, on peut observer des phénomènes allant justement dans des directions opposées. Par exemple, en France, qui a des bases fortes en droit public, l'obligation de la transposition des directives européennes en matière de service public ainsi que l'approche contractuelle de la réglementation communautaire font que la diminution de l'importance du droit de service public est à l'ordre du jour, ainsi que la mise en cause du bien-fondé de la réglementation spéciale (Bourdon, 2005, pp. 284-288; Wolikow, 2010, pp. 172-179; Touzeil-Divina, 2010, pp. 228-233). Toutefois, la "privatisation" du statut devrait aussi être évaluée d'une manière plus précise. Selon une étude comparée, en dépit du retrait de la réglementation spécifique, aucun pays n'a totalement délaissé le statut de droit public (Demmke et Moilanen, 2012, pp. 2-3). Néanmoins, le concept du statut uniforme est en voie de disparition, plusieurs catégories de statuts existent dans le droit public. La réglementation devient de plus en plus fragmentée. Le statut relevant du droit public se limite au noyau de la souveraineté de l'État, 
au personnel des ministères, de la police ainsi que de la justice, alors que l'importance du statut du droit public diminue dans le domaine de la santé, de l'enseignement, etc. Si la dichotomie du droit public/privé est présente dans presque chaque État membre, la proportion de personnel statutaire est en baisse. Dans les pays appartenant au système dichotome, l'agent public peut être recruté par nomination ou contrat dans le même emploi.

D’après moi, ces précisions ne changent pas la direction de l'évolution mais complètent plutôt une image complexe. En tout cas, il semble clair que l'avenir du droit public constitue un sujet primordial de débat professionnel dans presque tous les pays européens, comme en Hongrie.

\subsection{Contexte national}

À titre liminaire, penchons-nous sur le nombre d'agents travaillant dans la fonction publique au sens large. Ce chiffre était proche de 900000 en 2015. ${ }^{1}$ Plus exactement, en 2015, 886000 personnes ont obtenu un salaire qui provenait du budget public. En 2013, l'effectif des fonctionnaires en pourcentage de la population active était de 23,9\%. Cette proportion dépassait le taux moyen des pays de l'OCDE (OECD, 2015a, p. 85).

En 2012, en Hongrie, les dépenses des administrations publiques en pourcentage du PIB étaient de 48,7 \% ce qui dépassait la moyenne $(43,3 \%)$ des pays d'Europe centrale et orientale (PECO) et la moyenne de l'OCDE $(45,1 \%)$ (OECD, 2015b, p. 31). Cependant, ces dépenses montrent une tendance à la baisse. En effet, depuis le début de la crise financière, elles ont diminué de 2 points contrairement à la plupart des pays voisins ou à ceux de l'OCDE dont les dépenses publiques ont augmenté pendant la même période.

Les rémunérations en pourcentage des dépenses de l'administration publique, en 2012 ont baissé à 20,3\%, ce qui est semblable au pourcentage des pays voisins mais est inférieur à la moyenne de l'OCDE (OECD, 2015 b, p. 46). La diminution est imputable à plusieurs décisions. Depuis 2008, l'indexation salariale est gelée. Par ailleurs, plusieurs allocations ont été supprimées, dont la prime de fin d'année correspondant au salaire du mois de décembre. L'effectif de la fonction publique a également subi plusieurs baisses.

En Hongrie, la réglementation relative au statut des agents publics n'est pas uniforme, mais fortement différenciée. En effet, différentes lois règlementent les différentes catégories d'employés de la fonction publique. Par conséquent, les différents groupes d'agents publics se retrouvent dans des statuts distincts. Une dizaine de lois différentes codifient les différents statuts de la fonction publique mais aucun cadre réglementaire ne les harmonise. Du fait de cette fragmentation, la mobilité entre les différentes catégories des agents publics est fortement limitée. Toutefois, l'obstacle à la mobilité n'est pas seulement juridique. Les différentes rémunérations

1 Source: Ministère de l'Économie nationale (ci-après: MEN) 
et pratiques dans le domaine de ressources humaines (ci-après les RH), l'hétérogénéité des cultures d'organisations, les styles managériaux typiques à chaque statut, la diversité des exigences, ainsi que les multiples systèmes d'intéressement empêchent aussi la mobilité.

\section{Les objectifs de la réforme de la fonction publique hongroise}

En 2010, le gouvernement a entamé une réorganisation étatique dans le but de se rapprocher des objectifs d'un « État fonctionnel ». En effet, le gouvernement souhaitait non seulement modifier quelques éléments de l'administration publique mais aussi changer profondément le fonctionnement de l'État. Les réformes relatives à la justice, aux collectivités locales et à l'administration publique ont été entamées. Cette dernière touchait tous les éléments de l'administration publique, y compris la fonction publique. Elle suivait à la fois une approche stratégique et une approche complexe.

Ces deux approches devraient être mises en avant pour deux raisons. D'une part, en Hongrie, les grandes questions de la fonction publique ont été traitées sans approche stratégique pendant plusieurs cycles gouvernementaux. Cela a eu deux conséquences. Premièrement, les décisions concernant la politique de gestion du personnel ont souvent été soumises aux objectifs budgétaires du moment poursuivis par le gouvernement. Deuxièmement, les questions relatives à la réglementation ont été traitées en priorité au détriment des problèmes de gestion des ressources humaines. Par conséquent, la modernisation de la fonction publique s'est limitée à des modifications de la législation. D’autre part, en Hongrie, de manière générale, on peut relever une tendance à surestimer le rôle de la réglementation, à l'image du modèle russoallemand, doté traditionnellement d'une approche juridique. Néanmoins, le contexte social de la fonction publique change et la société exprime de plus en plus fort sa volonté que l'administration publique financée par les contribuables fonctionne le plus efficacement que possible. ${ }^{2}$

Compte tenu de l'importance de l'approche stratégique et complexe, le Programme de développement de l'administration publique "Zoltán Magyary » (ci-après: le Programme Magyary) a donc été adopté pour servir de base à la modernisation de la fonction publique. Selon le Programme Magyary, un État ne peut s'adapter rapidement et de manière flexible aux changements en mettant en avant les intérêts nationaux que si la fonction publique intègre non seulement les valeurs du modèle de carrière mais aussi les valeurs de la gestion moderne des ressources humaines fondées sur l'efficacité et la performance. Pour ce faire, il a défini comme objectif stratégique d'une part le développement d'une carrière prévisible et attractive, d'autre part la mise en place d'une gestion des ressources humaines basées sur l'emploi. Il a ainsi

2 Source: Ce changement s'explique aussi par le fait que le crédit budgétaire alloué aux ressources humaines représente 6,8 \% du PIB de la Hongrie. Ministère de l'Économie nationale (ci-après : MEN) (2013) 
défini en même temps deux directions de développement qui pourraient sembler contradictoires. L'une exige le renforcement du système de carrière tandis que l'autre suppose un système plus ouvert. Un système hybride est mis en place non seulement pour ce qui est du régime juridique de la fonction publique hongroise mais aussi pour certains statuts plus particuliers, ce qui devrait permettre de combiner des éléments appartenant au nouveau système, comme la flexibilité et la recherche de performance, avec les valeurs traditionnelles du système de carrière.

\section{3 "L'hybridation » du régime juridique}

\subsection{Statut des « fonctionnaires civils »}

La loi dite « Kttv. » est applicable aux « fonctionnaires civils » travaillant dans les ministères, aux services déconcentrés, aux collectivités locales, etc. En 2015, leur effectif était de 114000 personnes (12\% du secteur public). ${ }^{3}$ Ce sont eux qui exercent le pouvoir public au nom de l'État. La structure de leur statut ressemble à celui du système de carrière traditionnel. Leurs droits et obligations sont prévus par la « Kttv. » et autres règlements d'exécution, ce qui fait que le contenu de leur statut est défini par l'État de manière unilatérale, générale et impersonnelle (situation statutaire). Cela signifie que le législateur peut, à tout moment, changer les règles, modifier les droits et les obligations, et ceci sans le consentement des fonctionnaires. Ce mécanisme garantit également que l'emploi puisse être modifié conformément à l'évolution des intérêts du service ou de l'organisation (Bonnard, 1943, pp. 446-447). Ce point doit être d'autant plus souligné qu'il est dans l'intérêt général que le fonctionnement continu de l'administration publique soit assuré (principe de la continuité du service public) (Duguit, 1907, p. 416).

Cependant, la loi « Kttv. » a perdu beaucoup de caractères du système de carrière au cours des années, à la suite de la politique budgétaire d'austérité centrale, qui a rendu plus flexible le statut des «fonctionnaires civils». Cette évolution concernait les garanties d'emploi et la rémunération.

En ce qui concerne l'affaiblissement des garanties d'emploi, aux termes de la loi, non seulement le Parlement, mais aussi tout employeur, peut décider de la réduction des effectifs. Dans ce cas, les « fonctionnaires civils » peuvent être licenciés pour des raisons structurelles, par exemple la suppression de leur poste. À partir de 2011, une modification permet aux employeurs de licencier les «fonctionnaires civils » en raison d'une « perte de confiance ». Dans ce cas, «la flexibilisation » de la garantie de l'emploi n'est pas liée au maintien de l'équilibre du budget central mais au renforcement de "l'esprit de corps ». En effet, l'employeur ne peut en décider que s'il n'a plus confiance dans le « fonctionnaire civil ». Cette décision doit être motivée par un comportement qui porte atteinte aux valeurs professionnelles de l'employeur, par exemple

3 Source : MEN (2015) 
dans l'hypothèse où «le fonctionnaire civil» critique publiquement une décision quelconque émanant de son dirigeant.

La flexibilité peut être détectée même dans le domaine de la rémunération. À partir de 2001, une grande partie (au plus 30 \%) de la rémunération est fixée par l'employeur, en fonction du résultat de l'évaluation de la performance. Il convient de souligner que la Hongrie a instauré le système d'évaluation le plus rigoureux des pays de l'Europe de l'Est.

En 2006, plusieurs initiatives inspirées du concept de nouvelle gestion publique avaient été adoptées, mais ont été en grande partie abolies, en 2010, à la suite du changement de gouvernement et de l'adoption du Programme Magyary, inspiré par des idées néo-wébériennes. L'une d'entre elles prévoyait l'établissement d'une rémunération basée sur l'emploi. En 2008, un projet a été élaboré en vue de remplacer le système de traitement basé sur l'ancienneté de l'emploi, ce qui signifie que la rémunération du « fonctionnaire civil » ne se calculait pas en fonction de son classement mais du résultat de l'évaluation de son poste. Pour préparer la mise en place de ce projet, chaque emploi a été évalué auprès des ministères et intégrés dans un système de récompense basé sur le classement des emplois. Le projet a cependant échoué avant 2010 en raison du manque de soutien politique et de la résistance de la bureaucratie. Paradoxalement, l'idée du système de l'emploi s'est réincarnée dans la mise en place d'une gestion des ressources humaines basée sur l'emploi, prévue par le nouveau gouvernement dans le Programme Magyary.

En 2008, le gouvernement a instauré une nouvelle manière de recruter. En effet, la sélection des fonctionnaires aux postes de dirigeant a été centralisée. Seul un centre de gestion du personnel pouvait examiner les compétences de chaque candidat. Compte tenu des résultats de cette façon de procéder, une liste uniforme de classement des candidats a été établie pour les employeurs décidant des nominations. Ainsi, la sélection se faisait sur les compétences définies préalablement pour chaque poste à repourvoir. De plus, un concours a été prévu comme condition préalable au recrutement. En 2010, les deux modifications - la sélection basée sur les compétences et le concours - ont été supprimées. Ainsi, les employeurs gèrent actuellement leurs recrutements de nouveau de façon autonome, sans qu'aucune règle ne les contraigne à examiner la capacité des candidats. Par conséquent, pour l'instant, ils disposent d'un pouvoir discrétionnaire pour décider des nominations.

Ce n'est pas par un hasard si la Hongrie, quelques années après son adhésion à l'Union Européenne, a été classée parmi les pays « [...] qui ont progressé dans certains domaines de la gouvernance de la fonction publique mais dont la conformité aux principes européens a diminué dans d'autres ». En général, son système de fonction publique démontre un niveau intermédiaire de conformité aux principes européens d'administration (Meyer-Sahling, 2009, p. 6). 
En 2011, la loi «Kttv.» est devenue une réglementation dichotome. Une modification a confirmé qu'il était désormais possible de conclure un contrat de travail pour des missions non directement liées à l'exercice de prérogatives de pouvoir public, de compétences de direction, de contrôle et de surveillance. Elle a plafonné à 10 \% l'effectif pouvant être ainsi employé, tout en précisant que cette limite pouvait être dépassée dans des cas bien motivés avec l'autorisation du ministre chargé de la politique du personnel. Il s'ensuit que le personnel n'est pas homogène, il se compose d'agents statutaires et non statutaires. En tant qu'acte de droit public créant le rapport juridique du travail entre l'administration publique et l'individu, la nomination est prédominante dans la fonction publique. Cependant, le nombre de «travailleurs » employés par un contrat est de plus en plus important, même dans la fonction publique hongroise. Leur taux $(11,8 \%)^{4}$ semble élevé même s'il reste inférieur à celui des pays ayant un système de carrière $(17,2 \%){ }^{5}$

La progression du nombre d'agents contractuels peut être expliquée par la recherche de plus grandes flexibilités. C'est-à-dire que le statut basé sur le système de carrière ignore le marché du travail et ses contraintes, et n'est pas apte à faire coïncider les compétences des fonctionnaires aux exigences professionnelles des postes qu'ils occupent. En outre, le système de carrière limite la mobilité externe, et ne permet pas aux agents venant du secteur privé et arrivés à mi-carrière de s'intégrer dans la fonction publique. En raison de la rigidité du système de carrière, le classement des agents publics ne peut pas garantir, dans certains postes, et notamment dans les postes « intellectuels », un salaire compétitif, et ne récompense pas les hautes études spécialisées avec une rémunération plus élevée. Les salariés du secteur privé bénéficient d'une rémunération bien plus élevée (d'environ 100000 forints bruts) que les agents publics employés dans des postes similaires.

En résumé, le statut des "fonctionnaires civils» est en pleine mutation, sa réglementation basée sur le système de carrière devient de plus en plus " ouverte ", tant sur le plan du recrutement que du licenciement. Il n'est ni un pur système de carrière, ni un pur système d'emploi, mais un système hybride qui évolue vers le régime de droit privé. C'est le statut contractuel qui pourrait faire valoir une flexibilité de plus en plus importante, exigée par la politique.

\subsection{Statut des " employés publics »}

Une autre loi dite " Kjt. » règle le statut des « employés publics », qui sont des prestataires de services publics sans pouvoir public. Ce sont les employés qui travaillent dans les institutions financées par le budget public, comme les hôpitaux, écoles et musées, etc. La Kjt n'est applicable auxdites entités que si le pouvoir public, État ou collectivités locales, les gère. Le personnel des institutions détenues par l'Église ou les fondations n'est pas concerné, leur régime juridique relève du droit privé (Code du travail).

4 Source: MEN (2013)

5 P.ex., le service public français (DGAFP, 2012, p. 289) 
En 2015, l'effectif des «employés publics» rassemblait 457800 personnes (soit 51,7\% du secteur public). ${ }^{6}$ La réglementation qui régit cette question est différente de celle de la loi « Kttv. ». Si les règles de la loi « Kttv.» sont impératives pour les deux parties, l'employeur et le «fonctionnaire civil» ne pouvant y déroger par une quelconque convention, la loi « Kjt.», dans certains cas permet à l'employeur et à «l'employé public» de conclure une convention collective et ainsi de déroger à la loi. En outre, la «Kjt. » prévoit même l'application subsidiaire du Code du travail, c'est-à-dire que la réglementation du Code du travail fait partie ex lege du statut des " employés publics » à condition que la loi « Kjt. " n'exclue pas expressis verbis l'application du Code du travail. Ainsi, bien que le statut des "employés publics » soit défini unilatéralement par une loi, leur régime règlementaire est à la fois impératif et dispositif. Donc, il s'agit d'une réglementation mixte qui se trouve, à cheval entre la loi "Kttv.", qui se fonde exclusivement sur la réglementation publique unilatérale, et le Code du travail qui permet en général aux parties de définir librement le contenu de l'emploi par un contrat en respectant quelques règles fondamentales.

\subsection{Statut des " agents du maintien de l'ordre public "}

Une autre loi distincte, dite " Hszt. », s'applique aux " agents du maintien de l'ordre public », par exemple, les agents de police, les sapeurs-pompiers, le personnel pénitentiaire, etc.

Même si le Programme Magyary a prévu la mise en place d'un système d'emploi, le gouvernement voulait maintenir quelques éléments du système de carrière dans certains secteurs de la fonction publique. C'est-à-dire qu'il est d'autant plus nécessaire de sauvegarder les éléments du système de carrière que les valeurs traditionnelles wébériennes déterminent le fonctionnement du secteur. C'est la raison pour laquelle on a fait la différence entre le statut des «fonctionnaires civils» et celui des «agents du maintien de l'ordre public ». Pour les services du maintien de l'ordre public un système plus fermé a été adopté. Ses caractéristiques sont les suivantes :

- Il y a 5 catégories d'emploi, et les agents sont classés dans l'une des catégories en fonction de leur poste, mais leur avancement professionnel se déroule en fonction de leur ancienneté.

- Les conditions de leurs avancements sont fixées par la loi. Par exemple, l'agent est tenu de recueillir un certain nombre de points au cours de formations continues ou de réussir un examen pour obtenir un grade supérieur.

- Le recrutement de l'extérieur est fortement limité, ce qui veut dire que les postes vacants sont souvent pourvus en interne. Le concours est publié à plusieurs niveaux. Au premier niveau, le poste vacant est publié à l'intérieur de l'organisation. Si la procédure ne permet pas

6 Source : MEN 
de sélectionner un candidat, le cercle des personnes ciblées est élargi et le poste est proposé aux agents d'autres organisations du maintien de l'ordre public ainsi que de l'administration publique. Une personne extérieure n'ayant pas de statut en tant qu'agent du maintien de l'ordre public ne peut être recrutée à ce poste que si les deux sélections précitées ne conduisent pas à une nomination. L'avancement des dirigeants est plus fermé. Ils ne peuvent monter à un grade plus élevé que s'ils avaient déjà passé au moins 3 ans dans une fonction inférieure. Partant, une personne qui n'a pas d'expérience auprès des services du maintien de l'ordre public ne peut pas être nommée à un poste de dirigeant.

On peut dire que ce statut relève aussi du système mixte, toutefois il est plus proche du système de carrière.

Pour résumer, les lois dites "Kttv. », "Kjt.», "Hszt.» ne suivent pas le même modèle. La loi sur le statut des agents du maintien de l'ordre public se fonde plutôt sur le système de carrière, fermé, tandis que le statut des «fonctionnaires civils », et celui des « employés publics » sont plus proches du système ouvert, du droit de travail. Donc, "l'hybridation" se manifeste non seulement dans l'une ou l'autre loi mais aussi dans le régime juridique complet de la fonction publique où on peut distinguer les lois des statuts selon les modèles qu'elles suivent.

\section{La transformation de la gestion des ressources humaines}

L'évolution de l'effectif de la fonction publique montre qu'en dépit de plusieurs réductions du personnel, le nombre de fonctionnaires n'a quasiment pas changé. Pour trouver la raison de ce phénomène, les chercheurs ont mené une étude scientifique analysant de manière complexe l'effectif de la fonction publique, identifiant ainsi les caractéristiques du secteur des $\mathrm{RH}^{7}$ A la suite de l'analyse, un bilan des lacunes et des progrès a été établi par le biais des processus des $\mathrm{RH}$.

Le résultat de cette étude permet de constater qu'en Hongrie, de manière générale, la gestion des ressources humaines au sein des organisations étant fortement réglementée, elle est essentiellement dominée par l'administration et la bureaucratie. Les spécialistes des RH passent la plupart de leur temps, d'une part, à s'occuper de tâches juridiques et administratives portant sur le statut des fonctionnaires, d'autre part à fournir les données et les statistiques demandées par leur hiérarchie. Partant, ils sont chargés avant tout de résoudre les problèmes juridiques, administratifs, tandis qu'ils

7 C'était en 2013, dans le cadre de la réforme de la fonction publique, qu'a été prévue la possibilité d'effectuer des recherches pour examiner les changements survenus dans la gestion du personnel et trouver des possibilités d'amélioration de la gestion des ressources humaines. Les résultats des recherches sont contenus dans l'étude. Les données évoquées dans cette partie sont tirées de ces recherches. Lorsque ce n'est pas le cas, la source des données est signalée entre parenthèses. 
n'ont pas suffisamment de temps pour s'occuper des individus en tant que professionnels considérés sous l'angle des RH.

Les lacunes et les progrès enregistrés se présentent comme suit.

\subsection{La planification stratégique}

\section{Lacunes}

La gestion des ressources humaines manque d'approche stratégique. Là où la stratégie humaine existe, elle n'est pas toujours liée à la stratégie organisationnelle, ou à celui d'autres secteurs (financier, informatique, communication, etc.). Une gestion prévisionnelle des effectifs, des emplois et des compétences n'est pas mise en place. Les ministères n'ont pas d'appui technique pour gérer leur personnel à long terme. Ils ne disposent pas de statistiques appropriées et fiables pour mieux connaître quantitativement et qualitativement leurs fonctionnaires. Il manque aussi une instance gouvernementale chargée d'accompagner les ministères tout au long du processus de la planification et d'assurer la nécessaire coordination interministérielle. Le manque de « réflexion stratégique » est évoqué par une analyse de l'OCDE qui révèle aussi qu'en Hongrie, les pouvoirs publics n'ont quasiment pas recours aux instruments de la gestion stratégique (OECD, 2013, p. 97). Son index composite (0-1) dépasse à peine 0,1 tandis que la valeur moyenne de l'OCDE est de 0,5. Avec ce résultat, la Hongrie est au dernier rang des pays examinés.

\section{Progrès}

Certes, de manière isolée, il existe des organisations (par exemple quelques ministères) qui ont recours à des stratégies et processus humains et qui utilisent les fonctions humaines de façon intégrée. La planification s'y fait sur la base des compétences. Les fonctionnaires sont ouverts au changement, ils jugent que leur situation serait fondamentalement influencée et améliorée si la planification des forces de travail, la gestion de l'effectif, le développement des RH (formations) se faisaient sur la base d'un management tenant compte du rendement. De manière moins paradoxale, $60 \%$ des fonctionnaires en place dans le système de carrière se déclarent favorables à un système plus ouvert, ou plutôt à un système alliant les aspects positifs des systèmes ouvert et fermé.

\subsection{Système d'organisation du travail}

\section{Lacunes}

Le cahier des charges des postes n'est pas établi du tout, ou seulement de manière très lacunaire. Près de 40 \% des fonctionnaires ne sont renseignés précisément sur les tâches à accomplir qu'après leur nomination. Dans $30 \%$ des services, les dirigeants attendent plus, en qualité et en quantité, que ce qui est prévu dans la description des postes. L'utilisation des cahiers des charges 
est sporadique, et même si ceux-ci existe, ils sont incomplets. Par conséquent, il manque des profils de poste identiques qui correspondent aux différents services. Les formes de poste atypiques (travail à temps partiel, à distance, en temps partagé, etc.) ne sont introduites que marginalement. Les activités de monitoring et de contrôle en matière de $\mathrm{RH}$ sont quasiment inexistantes.

\section{Progrès}

L'analyse et l'évaluation des postes ont débuté. Jusqu'à présent, environ 15000 emplois ont été soumis à une évaluation. Une loi a été votée concernant les services de maintien de l'ordre public qui met en place un système de RH basé sur l'emploi. Une proposition similaire est en préparation pour les fonctionnaires civils. Les descriptions de poste sont devenues uniques. Le système d'organisation du travail fonctionne convenablement (temps de travail, temps de repos, congés).

\subsection{La gestion des flux}

\section{Lacunes}

Des planifications en matière d'effectif fonctionnent. Cependant, elles ne s'adaptent pas à la stratégie organisationnelle ou à la stratégie humaine, mais plutôt aux exigences sur une période d'un an, donc sur le court terme. Pour la planification, les éléments du marché (salaire, offre-demande) ne sont que très peu pris en considération, ce qui s'explique par la non-flexibilité du système de carrière.

Il est évident que le marché du travail influence la fonction publique, étant donné que c'est sur ce même marché que l'administration publique recrute de ses agents qualifiés. Toutefois, en Hongrie, selon certains auteurs, l'impact du marché du travail se manifeste différemment dans la fonction publique que dans le secteur concurrentiel. Ils disent que l'administration publique est obligée de composer avec un marché du travail dominé par l'offre, dans le sens où l'administration publique emploie en masse des personnes licenciées du secteur privé ou des personnes qui ne peuvent pas trouver un emploi dans le secteur privé. Selon lesdits auteurs, «l'administration publique accueille, en tout cas en partie, ces personnes, qu'elles aient, ou non, besoin d'elles, tout en leur payant une rémunération d'un niveau bien plus élevé que celui qui serait justifié » (Gajduschek, 2010, p. 25). "L'administration publique a perdu son avantage sur le secteur privé à cause de son régime des carrières et se trouve maintenant dans la position du perdant » (Gajduschek, 2010, p. 23).

En ce qui concerne l'évolution de la rémunération, on peut dégager deux tendances. La première montre que l'évolution du salaire net moyen de la fonction publique suit généralement celle du PIB. Si le PIB diminue, les salaires baissent aussi. Toutefois, la diminution du salaire net moyen de la fonction publique est plus importante que celle du PIB et que celle du salaire net moyen dans le secteur privé. Par exemple, entre 1989 et 1996 le salaire net moyen a baissé de presque $40 \%$ dans la fonction publique, tandis que 
le PIB et le salaire net moyen du secteur privé ne diminuaient respectivement que de $15 \%$ et $23 \%^{8}$. La deuxième tendance montre que si l'évolution du PIB s'est inversée et s'est mise à augmenter, le secteur public en a davantage profité que le secteur privé. Pourtant, entre 1996 et 2006, le salaire net a augmenté tant dans le secteur public que dans le secteur privé, le niveau du salaire net moyen du secteur public a rattrapé celui du secteur privé. Au cours des dernières années, la différence salariale entre secteur public et secteur privé est devenue de plus en plus importante. C'est la raison pour laquelle plusieurs décisions récemment prises visent à augmenter les rémunérations de certaines branches de la fonction publique, comme l'enseignement public ou le maintien de l'ordre public.

La concurrence de marché est jugée particulièrement négative dans le secteur du maintien de l'ordre public. En effet, le secteur privé attire de plus en plus les professionnels du secteur du maintien de l'ordre public. C'est la raison pour laquelle ce secteur fait face à une pénurie de jeunes. L'exode du secteur public a atteint un tel niveau qu'il empêche les organisations publiques d'accomplir leurs tâches de manière efficace.

Il n'existe pas de politiques et de modes de recrutement aboutis. En dehors des curriculum vitae, lettres de motivations et entretiens directs, les modes de recrutement plus ciblés ne sont pas utilisés (formulaire sur le trajet de vie, entretien structuré, test de stress, évaluation à 360 degrés, évaluation AC/DC et centre de développement, divers tests de compétence et de personnalité, examen des références).

Au sein de la plupart des organisations, l'intégration des nouveaux employés (apprentissage sur le lieu de travail) n'est pas réglementée et est laissée à la discrétion des dirigeants, qui déterminent la manière de procéder.

\section{Progrès}

C'est dans le cadre de ce processus que l'on constate le plus d'innovation et d'indicateurs de points de vue proactifs (existence de banques de données, renouvellement des cadres et des dirigeants, programmes d'entretien des talents). Dans ce secteur, on retrouve également des activités qui représentent, pour la plupart, des charges administratives considérables et sont assurées par des professionnels en matière de RH. Par exemple, on trouve la gestion des affaires juridiques et administratives portant sur la nomination, les départs au sein d'une administration ou les mouvements entre administrations, le détachement, la reconnaissance sur la base du mérite, la gestion des procédures disciplinaires et liée au dédommagement, la formation et la formation professionnelle, ainsi que la gestion de cette dernière, les changements de statuts, les questions relatives à la retraite.

8 Source: MEN 


\subsection{Le développement des $\mathrm{RH}$}

\section{Lacunes}

La formation continue est en grande partie financée par des fonds européens. Il s'ensuit qu'avec l'arrêt de nombreux financements européens, il faudra mobiliser des ressources nationales considérables. En ce qui concerne le développement du personnel directement lié au travail (angl. on-the-job), les progrès sont minimes. Le monitoring et le coaching sont à l'état embryonnaire. Par ailleurs, les données montrent qu'une part considérable des dirigeants accepterait d'accorder une place plus importante au coaching (maintien de l'ordre 19,6\%, administrations locales $40 \%$, administrations d'État 24,3\%).

Il manque des solutions de gestion de la connaissance et de partage du savoir entre les différentes entités administratives. Les programmes de période d'essai, les bourses ou autres stages ne sont pas suffisamment promus.

\section{Progrès}

De nombreuses innovations sont à noter pour ce qui est des formations extérieures au travail (angl. off-the-job). On note une progression considérable des différentes formes de développement indépendant de la connaissance : e-learning et blended learning. L'Université nationale de service public propose 300 formations professionnelles dont 223 se font en e-learning. Un système informatique destiné à la gestion de la formation professionnelle, nommé «Probono » permet non seulement de rechercher des formations, mais aussi aux administrations de préparer le plan annuel de formation des fonctionnaires, et donc l'exécution de ce plan.

\subsection{La gestion de la performance}

\section{Lacunes}

La culture de l'évaluation est rudimentaire. L'évaluation de la performance est considérée comme une obligation juridique formelle. Environ la moitié des organes de l'administration publique gère les évaluations de performance uniquement sur papier. Cela signifie que les fonctionnaires reçoivent des formulaires d'évaluation pré-remplis, sans discussion préalable, ou pire encore, ils se les remplissent eux-mêmes. L'évaluation à 360 degrés n'est pas utilisée. L'évaluation de groupe ou d'une administration tout entière est peu utilisée. À cause des changements rapides de la structure administrative et de son fonctionnement, il est impossible de prévoir, même un an à l'avance, des objectifs de performance. Par ailleurs, les dirigeants sont dans l'incapacité de prévoir chaque année de nouveaux objectifs pour chaque tâche et chaque poste particulier. L'individualisation des objectifs à poursuivre au niveau des organes est un élément particulièrement problématique pour les dirigeants.

Les administrations n'utilisent que sporadiquement des instruments de mesure et indicateurs de performance. Les évaluations ne sont pas mises en relation de manière adaptée avec le développement, la motivation 
et la gestion de carrière. Les résultats des examens de satisfaction internes et les enquêtes d'opinion sont à peine utilisés pour développer les administrations, transformer la culture organisationnelle ou évaluer le niveau des activités de direction. Cependant, les administrations de maintien de l'ordre public constituent une exception car ils réalisent des enquêtes d'opinion ayant trait au jugement que porte la société sur la police.

\section{Progrès}

En 2002, l'évaluation de la performance individuelle au sein de la fonction publique a été lancée. Grâce à cette initiative, de nombreuses expériences positives ont déjà été obtenues. Pour certains secteurs, l'évaluation de la performance basée sur la compétence a déjà eu lieu dès le lancement de ce système et cette initiative a été élargie à l'ensemble de la fonction publique en 2006. L'évaluation de la performance est devenue régulière dans la gestion des RH. La majorité du personnel accepte et juge les évaluations nécessaires. Certaines organisations de l'administration publique ont commencé à mettre en place des évaluations de groupe et d'organes tout entier.

Pour conclure, la performance de la fonction publique ne peut être améliorée que si le remaniement de l'administration publique est accompagné du renouvellement de la gestion des $\mathrm{RH}$. Par conséquent, la réserve d'efficacité du système réside essentiellement non pas dans la réduction de l'effectif, mais dans la mise en place d'une gestion intégrée des ressources humaines, basée sur l'emploi et la stratégie, qui permette de transformer les opportunités concernant l'intégration organisationnelle en des résultats concrets.

\section{Conclusion}

Pour résumer, on peut dire que la fonction publique hongroise est en train de se transformer profondément. Les grandes questions de la fonction publique sont abordées selon une approche stratégique. Le Programme Magyary sert de base à sa modernisation. Les changements prévus touchent à la fois la réglementation et la gestion des ressources humaines. Les préoccupations liées à l'adoption de techniques de RH empruntées au secteur privé et au placement de l'emploi au centre de la gestion des ressources humaines, se mettent à transformer le régime juridique basé traditionnellement sur la carrière. La sauvegarde des valeurs traditionnelles reste toutefois une préoccupation. Pour satisfaire à la fois les exigences de plus grande flexibilité et de sauvegarde des valeurs traditionnelles, deux initiatives ont été prises: d'une part, la mise en place d'un système hybride qui intègre à la fois des éléments de flexibilité et de performance, et le maintien, du moins en partie, des principes de la carrière, et d'un autre côté la gestion des ressources humaines basées sur l'emploi. 
Pour atténuer les effets négatifs des cloisonnements consécutifs à la fragmentation de la réglementation, d'autres orientations de la réforme de la fonction publique visent à promouvoir la mobilité transversale.

Le remaniement de l'administration publique ne peut être efficace que s'il est accompagné par une gestion intégrée des ressources humaines fondée sur la stratégie.

Une approche stratégique, une flexibilité et une mobilité accrues, une gestion stratégique basée sur l'emploi permettraient d'accroître la compétitivité de la fonction publique.

Dr. Zoltán Hazafi, PhD, professeur agrégé, chef de l'Institut des Ressources Humaines à la Faculté de la science de l'État et de l'Administration Publique de l'Université Nationale de Service Public (Hongrie)

Dr. Hazafi, juriste, ancien élève de l'ENA, enseigne plusieurs cours en matière de la fonction publique aux différents niveaux de l'enseignement (bachelor, master et $P h D)$. Ses recherches incluent les différentes dimmensions de la fonction publique, comme par exemple l'élvolution du droit de la fonction publique, l'adaptation des moyens de la gestion des ressources humaines, utilisés déjà avec succès dans le secteur privé ou la comparaison les tendances de l'évolution de la fonction publique dans le domaine international. Il est le membre de Comité de Rédaction de 2 journaux: "Pro Publico Bono - l'Administration Publique Hongroise » et "Nouvelle Adminstration Publique Hongroise ». 


\section{Références}

Berthelemy, H. (1933). Traité élémentaire de droit administratif(13e éd.). Paris.

Bonnard, R. (1942). Précis de Droit administratif (4e éd.). Paris.

Bourdon, J. (2005). Vers une banalisation du droit de la fonction publique? AJFP Actualités Juridiques, Fonctions Publiques, $\left(n^{\circ} 6\right)$, 284-288.

Chemla-Lafay, A., Deleplace, M.-T., le Flécher, C., Meimon, J. et Trosa, S. (2008). Performance de la fonction ressources humaines: définitions et cadre d'analyse. Département Recherche, Études, Veille - Institut de la gestion publique du développement économique, ministère du Budget, des Comptes publics et de la Fonction publique. Repéré à http://infos.emploipublic.fr/wp-content / uploads/2011/11/rapport_chemla_lafay.pdf

Demmke, C. et Moilanen, T. (2012). The future of public employment in central public administration restructuring in times of government transformation and the impact on status development. European Institute of Public Administration.

DGAFP. (2012). Rapport annuel sur l'état de la fonction publique (Politiques et pratiques de ressources humaines, Faits et chiffres). Paris : La documentation française.

Duguit, L. (1907). Droit constitutionnel (1re éd). Paris.

Gajduschek, G. (2010). Gestion de la main d'œuvre pilotée par l'offre ? Ou qu'estce qui détermine la composition du personnel des fonctionnaires ? (rédigé en hongrois). Új Magyar Közigazgatás, (8), 14-26.

Gouldner, A. W. (1953). Patterns of Industrial Bureaucracy. New York, NY: Glancoe, Free Press.

Hazafi, Z. (2014). Le nouveau modèle de carrière dans la fonction publique - les dimensions juridiques et de la gestion des ressources humaines du développement de la fonction publique. Budapest : Magyar Közlöny Lap- és Könyvkiadó. Repéré à http://magyaryprogram.kormany.hu/download/d/fa/ a0000/_ZaroTanulmany_FR_online_AROP2217.pdf

Jeze, G. (1930). Principes généraux du droit administratif (3e éd.) Paris : Dalloz.

Lorincz, L. (1981). La relation de l'administration publique à l'économie et à la politique (rédigé en hongrois). Budapest : Közgazdasági és Jogi Könyvkiadó.

Magyary, Z. (1942). L'Administration publique en Hongrie (rédigé en hongrois). Budapest : Királyi Magyar Egyetemi Nyomda.

van der Meer, F. M., van der Berg, C. F. et Dijkstra, G. S. A. (2013) Repenser le "marché bureaucratique » : l'évolution du statut (juridique) des fonctionnaires en Europe. Revue Internationale des Sciences Administratives, 79(1), 95-113. doi:10.3917/risa.791.0095

Meyer-Sahling, J.-H. (2009). Durabilité des réformes de la fonction publique en Europe centrale et orientale cinq ans après l'accession à l'UE. Document SIGMA (No. 44). 10.1787/5k40htrhpbtd-fr

Moniolle, C. (2010). Droit de la fonction publique et gestion des ressources humaines : entre complémentarité et opposition. AJFP Actualité Juridique, Fonctions Publiques, ( $\left.n^{\circ} 5\right), 234-240$.

OECD. (2013). Gestion stratégique des ressources humaines. Dans Panorama des administrations publiques 2011. Paris: OECD Publishing. doi:10.1787/gov_glance-2011-22-fr

OECD. (2015a). Government at a Glance 2015. Paris : OECD Publishing. doi:10.1787/gov_glance-2015-en 
Transition d'un système de carrière à système d'emploi au travers d'un modèle hybride

(Modernisation de la fonction publique en Hongrie)

OECD. (2015b). Kormányzati körkép: Hogyan teljesít Magyarország? Paris : OECD Publishing. doi:10.1787/9789264233720-en

Salon, S. et Savignac, J. C. (2013). Code de la fonction publique commenté. Paris : Dalloz-Sirey. Repéré à http://www.dalloz.fr/documentation/ Document?id=CDFP

Touzeil-Divina, M. (2010). "Travaillisation » ou « privatisation » des fonctions publiques? AJFP Actualités Juridiques, Fonctions Publiques, ( $\left.n^{\circ} 5\right), 228-233$.

Vedel, G. et Delvolvé, P. (1958). Droit administratif. Paris : Presse Universitaire de France.

Wolikow, J. (2010). Fonctionnaires et salariés : différences, convergences. AJFP Actualités Juridiques, Fonctions Publiques, ( $\left.n^{\circ} 4\right), 172-179$. 
POVZETEK

1.02 Pregledni znanstveni članek

\section{Prehod s kariernega sistema na sistem zaposlovanja prek hibridnega modela (posodobitev javne službe na Madžarskem)}

Posodobitev madžarske javne službe, ki se je začela leta 2010, temelji na zapletenem strateškem pristopu ter na kompromisu med starimi in novimi vrednotami. Strateška cilja sta razvoj predvidljive in privlačne kariere na eni strani ter vzpostavitev upravljanja človeških virov na podlagi zaposlovanja na drugi. Vrednote kariernega modela in sodobnega upravljanja človeških virov se na prvi pogled morda zdijo protislovne. Vendar pa lahko hibridni sistem, ki je značilen za pravno ureditev madžarske javne službe, združuje različne vrednote, tako da zaposlovanje postavlja v središče upravljanja človeških virov. Poleg tega »hibridizacija« ni le ena od posebnosti madžarske javne službe, ampak tudi posebnost spreminjajočega se mednarodnega okolja. 